myjournal manuscript No.

(will be inserted by the editor)

\title{
Generation of long-living entanglement using cold trapped ions with
}

\section{pair cat states}

\author{
M. Abdel-Aty \\ Mathematics Department, College of Science, Bahrain University, 32038, Kingdom of Bahrain
}

Received: date / Revised version: date

\begin{abstract}
With the reliance in the processing of quantum information on a cold trapped ion, we analyze the entanglement entropy in the ion-field interaction with pair cat states.
\end{abstract}

We investigate a long-living entanglement allowing the instantaneous position of the center-of-mass motion of the ion to be explicitly time dependent. An analytic solution for the system operators is obtained. We show that different nonclassical effects arise in the dynamics of the population inversion, depending on the initial states of the vibrational motion. We study in detail the entanglement degree and demonstrate how the input pair cat state is required for initiating the long living entanglement. This long living entanglement is damp out with an increase in the number difference $q$. Owing to the properties of entanglement measures, the results are checked using another entanglement measure (high order linear entropy).

\section{Introduction}

Controlling quantum systems and the couplings between various quantum degrees of freedom is one of the major goals in quantum information processing [1]. In the early days of quantum optics, the dynamics and behavior of typical quantum optical systems, such as the laser, was dominated by dissipative effects and uncontrolled fluctuations of system parameters. Systems of single trapped ions 2 3,4 and single atoms strongly interacting with a high-Q cavity were developed, corresponding to single quantum systems which can be prepared microscopically and observed under conditions close to idealized and fundamental theoretical models [5]. By combining the technologies of ion trapping and cavity quantum electrodynamics, deterministic coupling of single ions to an optical field has been achieved 6 .

There is currently a diverse array of research interests being united under the banner of quantum information science, with fundamental research, in both experimental 
and theoretical arenas, providing new insights on different time dependent interaction models $7,8,9$. . Of great interest to physicists is the renewed vigor with which questions regarding the basic understanding and interpretation of quantum entanglement are being pursued. Also, the development of quantum information science and technology carries the potential of revolutionary impact on many aspects [10], as evidenced already by the applications in quantum cryptography, quantum communication, and rudimentary quantum computing. Entanglement is today considered a fundamental resource in nature when it comes to quantum computation and information 11], and measures of entanglement has become a major field of research [12].

Despite much effort and spectacular advances from several groups in recent years $13,14,15,16,17,18,19,20]$, long surviving entanglement remain elusive in the ionfield based systems. It is in this light that this paper will focus on long-living entanglement. The main motivation for the present work is its relevance to the field of quantum information, which is attracting broad interest in view of its fundamental nature and its potentially revolutionary applications. Our main purpose is twofold, first we wish to demonstrate how a pair cat state affect the entanglement for the ion-field interaction, and second we wish to see how the time-dependent amplitude of the irradiating laser field affect this entanglement. The improved understanding of entanglement has attracted much attention recently from the quantum information community which may lead to new insights into the physics of correlated systems in quantum optics. Therefore there is a great need to compute these in an efficient way. In particular, we consider a single trapped ion which can be laser cooled to the ground state of the trapping potential and discuss the roles played by the initial state setting and time-dependent amplitude of the laser field on the entanglement.

The paper is structured as follows: Section 2 is devoted to a brief description of the pair cat state and show that the quadrature distribution reflects the entanglement. In section 3 , we present the model of the completely quantized system and obtain an exact analytical solution of system operators. In section 4, we briefly discuss those features concerning the atomic inversion, which are relevant for collapse-revival phenomena. In section 5 , we analyze in detail how the pair cat state affect the general features of von Neumann entropy (a measure of the ion-field entanglement). This gives us the opportunity to stress the essential role played by pair cat states in this context and study the existing of a long living entanglement. We summarize our results at the end of the paper and make some conclusions.

\section{Pair cat states}

Superpositions of orthogonal states which exhibits macroscopic features appear to be of fundamental importance in recent studies of the foundations of quantum mechanics [21,22]. Superposition refers to a quantum system 
existing simultaneously in multiple states. This is usually told as the parable of Schrodinger's cat, shut up in a box with a vial of cyanide that at any moment might be triggered to release its deadly gas by a radioactive decay reaction. Resonant microcavities can be used to study the behavior of mesoscopic superposition coherent states. Some success has been reported in creating such superposition states within high Q cavities in the optical domains 23$]$.

Recently, schemes have been presented for the preparation of two-mode motional states of a trapped ion, such as pair coherent states [24, pair cat states [25], two-mode $\mathrm{SU}(1,1)$ intelligent states [26], and $\mathrm{SU}(2)$ cat states [27. These schemes operate in two-dimensional isotropic traps. In terms of conventional coherent states parameterized in terms of a complex number $\alpha$ [21], the Schrödinger cat state has the following form

$$
\left|S_{c a t}\right\rangle \equiv \frac{1}{\sqrt{2+2 \cos \phi \exp \left(-|\alpha|^{2}\right)}}\left(|\alpha\rangle+e^{i \phi}|-\alpha\rangle\right),
$$

where $|\alpha\rangle$ is a coherent state of amplitude $\alpha$, and $\phi$ is a real local phase factor. Note that the relative phase $\phi$ can be approximately controlled by the displacement operation for a given cat state with $\alpha>>1$ [28,29]. A special set of coherent states of the Barut-Girardello type known as pair coherent states has been formulated [30. If $\widehat{a}^{\dagger}(\widehat{a})$ and $\widehat{b}^{\dagger}(\widehat{b})$ denote two independent boson creation (annihilation) operators, then $\widehat{a} \widehat{b}\left(\widehat{a}^{\dagger} \widehat{b}^{\dagger}\right)$ stands for the pair annihilation (creation) operator for the two modes. The
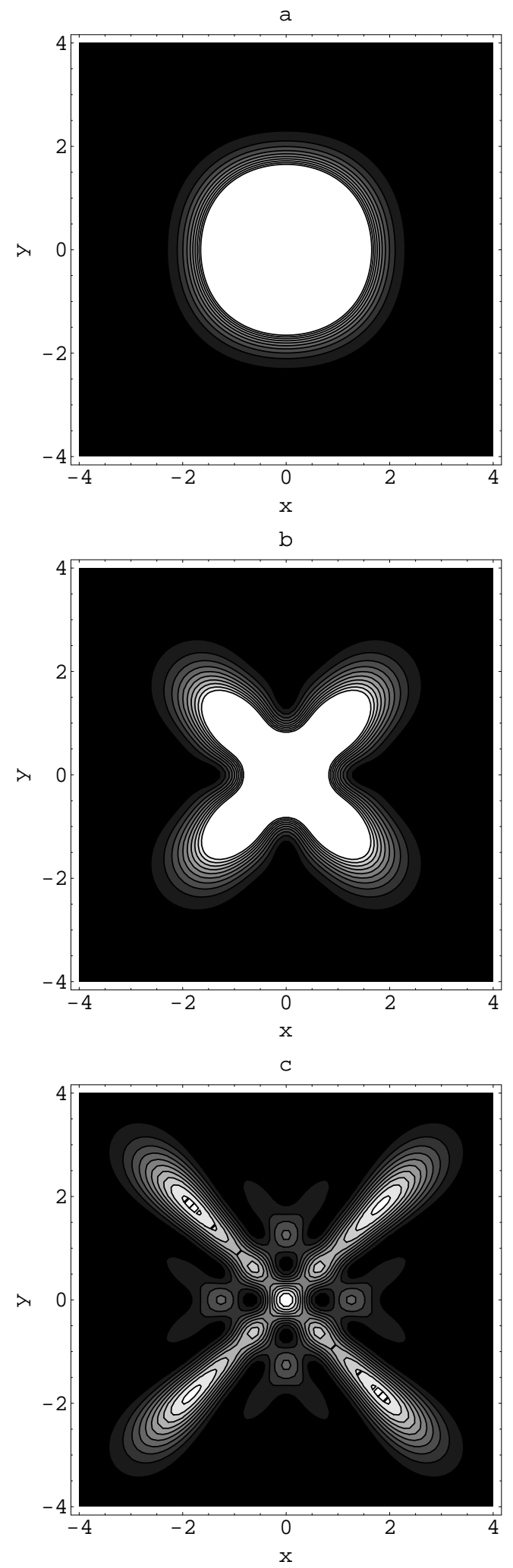

Fig. 1 Contour plot of the quadrature distribution function $P(x, y)$ for the pair cat state for $q=0, \phi=\pi / 2$ and for different values of the parameter $\xi$ where, (a) $\xi=0.1$, (b) $\xi=$ 1 and (c) $\xi=3$. 
pair coherent states $|\xi, q\rangle$ are defined as eigenstates of both the pair annihilation operator $\widehat{a} \widehat{b}$ and the number difference operator $\widehat{a}^{\dagger} \widehat{a}-\widehat{b}^{\dagger} \widehat{b}$, i.e.,

$$
\widehat{a} \widehat{b}|\xi, q\rangle \equiv \xi|\xi, q\rangle, \quad\left(\widehat{a}^{\dagger} \widehat{a}-\widehat{b}^{\dagger} \hat{b}\right)|\xi, q\rangle=q|\xi, q\rangle
$$

where $\xi$ is a complex number and $q$ is the charge parameter, which is a fixed integer. Furthermore, the pair coherent states can be expanded as a superposition of the two-mode Fock states,

$$
|\xi, q\rangle \equiv N_{q} \sum_{n=0}^{\infty} \frac{\xi^{n}}{\sqrt{n !(n+q) !}}|n, n+q\rangle,
$$

where $N_{q}$ is the normalization constant and can be written as $N_{q}=\left[|\xi|^{-q} J_{q}(2|\xi|)\right]^{-\frac{1}{2}}$, where $J_{q}$ is the modified Bessel function of the first kind of order $q$. Pair coherent states are regarded as an important type of correlated two-mode state, which possess prominent nonclassical properties such as sub-Poissonian statistics, correlation in the number fluctuations, squeezing, and violations of Cauchy-Schwarz inequalities 31,32. The pair cat states are defined as a superposition of two pair coherent states separated in phase by $180^{\circ}$ [33,

$$
|\xi, q, \phi\rangle \equiv N_{\phi}(|\xi, q\rangle+\exp (i \phi)|-\xi, q\rangle)
$$

where the normalization factor $N_{\phi}$ is given by

$$
N_{\phi}=\frac{1}{\sqrt{2}}\left(1+N_{q}^{2} \cos \phi \sum_{n=0}^{\infty} \frac{(-1)^{n}|\xi|^{2 n}}{n !(n+q) !}\right)^{-\frac{1}{2}} .
$$

It has been shown that these cat states may exhibit stronger nonclassical effects than the corresponding pair coherent states. The correlated two-mode cat state $|\xi, q, \phi\rangle$ is the eigenstate of the operators $\widehat{a}^{2} \widehat{b}^{2}$ and $\widehat{a}^{\dagger} \widehat{a}-\widehat{b}^{\dagger} \widehat{b}$
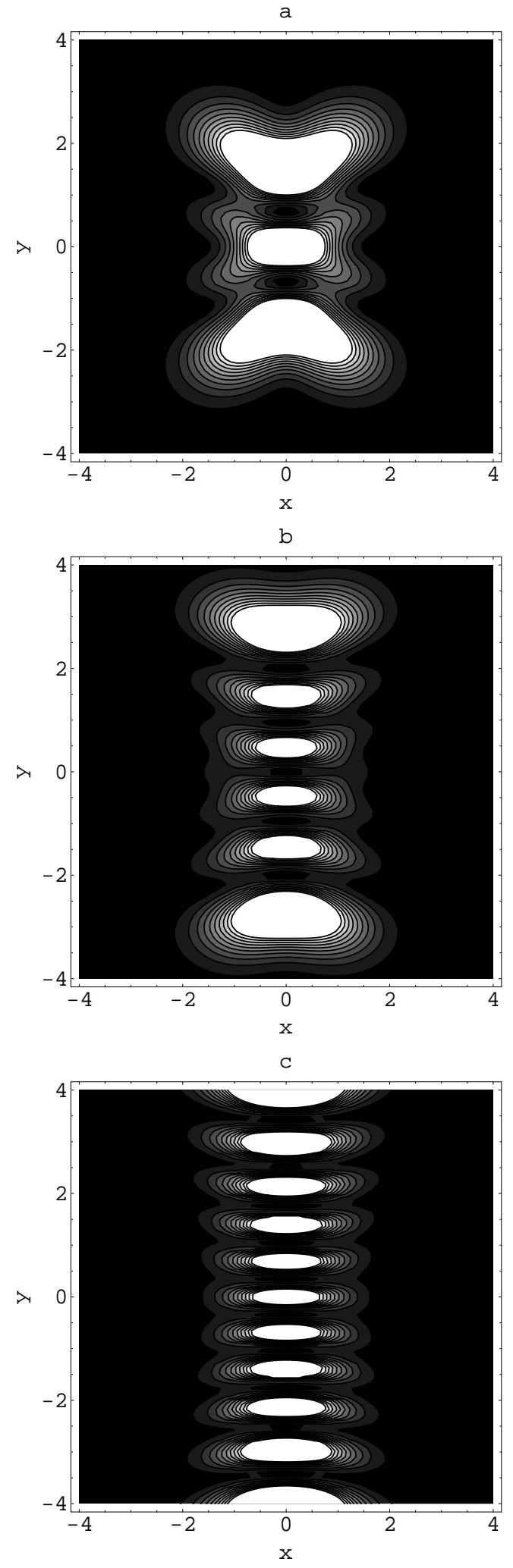

Fig. 2 Contour plot of the quadrature distribution function $P(x, y)$ for the pair cat state for $\xi=1, \phi=\pi / 2$ and for different values of the parameter $q$ where, (a) $q=2$, (b) $q=5$ and (c) $q=10$. 
with eigenvalues $\xi^{2}$ and $q$, respectively. In order to generate such cat states for the motion in a two-dimensional anisotropic trap we require two laser beams. Due to the strong nonclassical nature of the two-mode cat states, the generation of such states is of interest in testing quantum mechanics.

The coordinate space wave function is given by

$$
\begin{aligned}
\langle x, y \mid S\rangle & =N_{\phi}(\langle x, y \mid \xi, q\rangle+\exp (i \phi)\langle x, y \mid-\xi, q\rangle) \\
& =N_{\phi} \sum_{n=0}^{\infty}\left(1+(-1)^{n} e^{i \phi}\right)\langle x \mid n+q\rangle\langle y \mid n\rangle,(6)
\end{aligned}
$$

where $\langle x \mid n\rangle$ is a harmonic oscillator wave function. In this case, the quadrature distribution is given by

$$
\begin{aligned}
P(x, y)= & \left|N_{\phi}\right|^{2} \mid \sum_{n=0}^{\infty} \frac{\left(1+(-1)^{n} e^{i \phi}\right)}{\pi} \frac{2^{-(2 n+q)}}{n !(n+q) !} \\
& \times\left. H_{n+q}(x) H_{n}(y) e^{-0.5\left(x^{2}+y^{2}\right)}\right|^{2} .
\end{aligned}
$$

For fixed values of the parameter $q$ the periodic nature in the quadrature distribution function in the different values of the parameter $\xi$ are shown in figure 1 , where, we plot $P(x, y)$ for $\xi=0.1,1$ and 3 . It is shown that the regions of the quadrature distribution in the $(x, y)$ plane are symmetric with respect to $y=-x$ and with respect to $y=x$ (figures $1 \mathrm{~b}$ and $1 \mathrm{c}$ ). It is clear that the present distribution reflects the entanglement present in the two-mode pair cat states. The main curiosity is that, if squeezing is strong enough, the ring-like quadratic distribution function collapses to a quasi one dimensional object with a cigar form (see figure 1a).

The origin of such behavior is in the generation of pair cat states in the microscopic regime, where the con- tributions of the different components of the pair cat states are located close to the phase space origin, competing with each other. When the values of the parameter $\xi$ is increased, the shape of the quadrature distribution becomes more pronounced by involving multi-peak structure (symmetric peaks). This is in a good agreement with the general behavior of the quadrature distribution of the pair coherent state 34 . It is interesting mentioning here that, the two-mode pair cat states exhibit strong nonclassical features due to the correlation between the two modes.

In figure 2, we plot the quadratic distribution for different values of the number of quant $q$, (say $q=2,5$ and 10). We have shown that the behavior of the quadratic distribution makes possible, for sufficient small values of the indicator parameter $q$, to transform the multi-parts windows to a one circular part. This is a consequence of the intrinsic properties of the pair cat state. Nevertheless, perfect purification is not achieved in this case, compared with the case in which small value of $\xi$ is considered (see figure 1a and 2a). In figure 1a the window is not a two parts function, but is very narrow so that the conditional moment is approximately equal to one circle, this behavior may be obtained if the parameter $q$ takes smaller values. Note that the quadratic distribution is dramatically different from the case when we consider large values of $q$ (see figure $2 \mathrm{a}$ and $2 \mathrm{~b}$ ), and indeed here it is not possible to observe separate parts of the distribution. It is interesting to show in this figure that the 

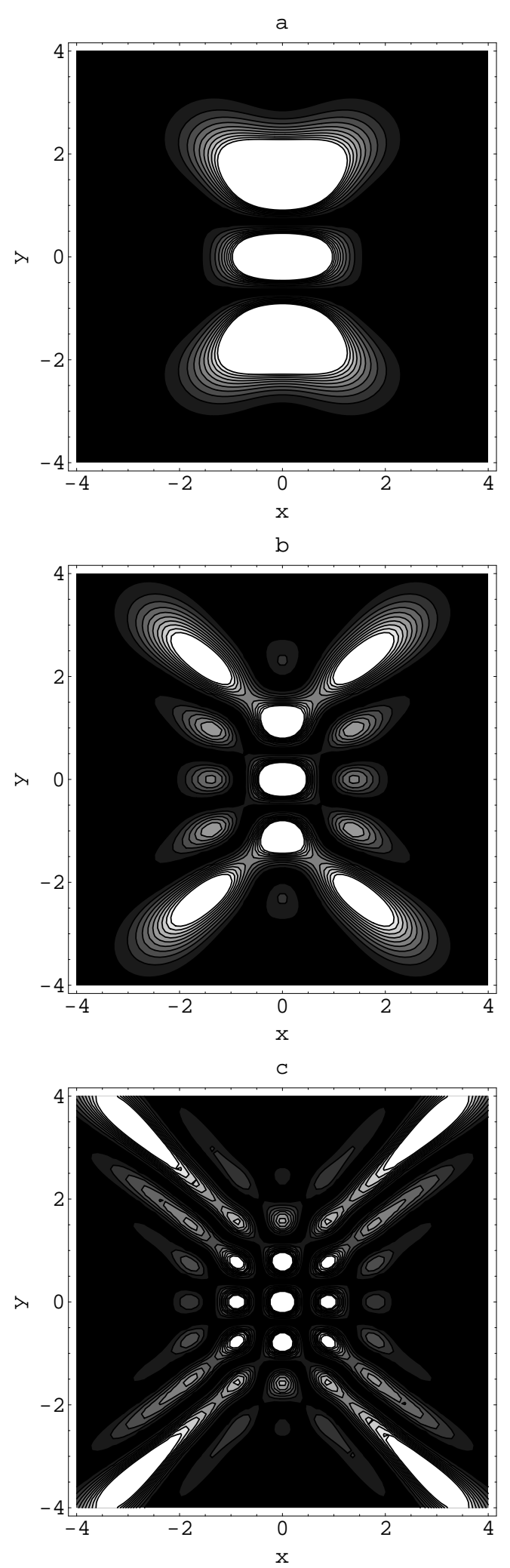

Fig. 3 Contour plot of the quadrature distribution function $P(x, y)$ for the pair cat state for $q=2, \phi=0$ and for different values of the parameter $\xi$ where, (a) $\xi=1$, (b) $\xi=3$ and (c) $\xi=7$ regions of the quadrature distribution in the $(x, y)$-plane are symmetric with respect to $y=0$ and with respect to $x=0$ (figures $2 \mathrm{~b}$ and $2 \mathrm{c}$ ).

In figure 3 , we set the parameter $\phi=0$ and take different values of $q$ and $\xi$. In this figure we show the evolution of $P(x, y)$ and its responses in a type of plot similar to that shown in figures 1 and 2 . It is shown that the regions of the quadrature distribution are symmetric with respect to $x=0$ and with respect to $y=0$ (figures $3 \mathrm{~b}$ and $3 \mathrm{c}$ ), but the general feature of this case is quit complicated compared with the previous cases. This can be understood from looking to equation (5), where, when we set $\phi=0$ this allows the contribution of the second term in equation (5). Some of the differences are seen in the contour interval if a very small change of the controller parameters $(\xi$ and $q$ ) is considered. This can be demonstrated from a contour plot, such as shown in figure 3 .

\section{Physical system and model Hamiltonian}

In the interest of retaining as much clarity as possible, we first recall some well-known facts about the system we wish to treat here, which consists of a single trapped ion interacting with a laser field 24]. We consider the situation where oscillating classical electromagnetic fields propagating in two-dimensional trap irradiate a single trapped ion. To see that let us consider the laser frequency is tuned to the frequency difference between the two modes of the trap. Consequently the Hamiltonian 
for such a system is given by 24, 25 26,

$$
\hat{H}=\hat{H}_{F}+\hat{H}_{A}+\hat{H}_{i n}
$$

where

$$
\begin{aligned}
\hat{H}_{F} & =\hbar \omega_{1} \hat{a}_{1}^{\dagger} \hat{a}_{1}+\hbar \omega_{2} \hat{a}_{2}^{\dagger} \hat{a}_{2}, \\
\hat{H}_{A} & =\frac{\hbar \omega_{0}}{2} \hat{\sigma}_{z}, \\
\hat{H}_{\text {in }} & \left.=-\wp \cdot E=-\wp \cdot \varepsilon(v t)\left[\exp \left[i\left(k_{1} \hat{x}+k_{2} \hat{y}-\omega t\right)\right)\right]+H . c .\right],
\end{aligned}
$$

with $\wp$ the dipole matrix element of the ion $\wp=\wp^{0}\left(\widehat{\sigma}_{+}+\right.$ $\left.\widehat{\sigma}_{+}\right), \varepsilon(v t)$ is a modulated amplitude of the irradiating laser field. We denote by $\omega_{i},(i=1,2)$ the fields frequencies, $\omega_{0}$ the natural frequency of the ion, and $v$ denotes the ionic velocity. Here the $\sigma^{\prime} s$ are the usual $2 \times 2$ Pauli matrices satisfying

$$
\left[\hat{\sigma}_{z}, \hat{\sigma}_{ \pm}\right]= \pm 2 \hat{\sigma}_{ \pm},\left[\hat{\sigma}_{+}, \hat{\sigma}_{-}\right]=\hat{\sigma}_{z} .
$$

We denote by $\hat{a}_{i}$ and $\hat{a}_{j}^{\dagger}$ the Bose operators for the quantized field mode which obey $\left[\hat{a}_{i}^{\dagger}, \hat{a}_{j}\right]=\delta_{i j}$, where $\delta_{i j}=1$ if $i=j$ and $\mathbf{0}$ otherwise. The operators $\hat{x}$ and $\hat{y}$ are the center of mass position of the ion which are quantized in the forms $\hat{x}=\Delta x\left(\hat{a}_{1}^{\dagger}+\hat{a}_{1}\right), \hat{y}=\Delta y\left(\hat{a}_{2}^{\dagger}+\hat{a}_{2}\right)$ and $\Delta x=\sqrt{1 /\left(2 v_{x} M\right)}, \Delta y=\sqrt{1 /\left(2 v_{y} M\right)}$ with $M$ being the mass of the trapped ion.

Making use of the a special form of Baker-Hausdorff theorem [35] the operator $\exp \left[i \eta\left(\hat{a}^{\dagger}+\hat{a}\right)\right]$ may be written as a product of operators i.e.

$$
\begin{aligned}
e^{i \eta\left(\hat{a}^{\dagger}+\hat{a}\right)} & =\exp \left(\frac{\eta^{2}}{2}\left[\hat{a}^{\dagger}, \hat{a}\right]\right) \exp \left(i \eta \hat{a}^{\dagger}\right) \exp (i \eta \hat{a}) \\
& =e^{-\eta^{2} / 2} \sum_{n=0}^{\infty} \frac{(i \eta)^{n} \hat{a}^{\dagger n}}{n !} \sum_{m=0}^{\infty} \frac{(i \eta)^{m} \hat{a}^{m}}{m !}
\end{aligned}
$$

Using equation (10), the interaction part of equation (8) can be written as

$$
\begin{aligned}
\hat{H}_{i n}= & -\wp^{0} \cdot \varepsilon(v t) \eta_{1} \eta_{2} \exp \left[-\frac{1}{2}\left(\eta_{1}^{2}+\eta_{2}^{2}\right)\right] \\
& \times \sum_{l=0}^{\infty} \sum_{m=0}^{\infty} \frac{\hat{a}_{1}^{\dagger l} \hat{a}_{1}^{l} \hat{a}_{2}^{\dagger m} \hat{a}_{2}^{m}}{(l+1) !(m+1) !}\left(i \eta_{1}\right)^{l}\left(i \eta_{2}\right)^{m} \\
& \times\left(\hat{a}_{1}^{\dagger} \hat{a}_{2}+\hat{a}_{2}^{\dagger} \hat{a}_{1}\right)\left(\hat{\sigma}_{-}+\hat{\sigma}_{+}\right),
\end{aligned}
$$

with $\eta_{1}=k_{1} \Delta x$ and $\eta_{2}=k_{2} \Delta y$. When we take LambDicke limit and apply the rotating wave approximation then the effective interaction Hamiltonian (10) takes the two photon bimodal interaction form. However, provided we adjust the strength of the electric field to include the time dependent factor $\varepsilon(v t)$. In this context we may refer to the work given in Ref. [22]. In this work the authors considered the usual JCM taking into account the effects of stochastic phase fluctuations in the atom-field coupling coefficient. This is achieved by modulated the coupling parameter to include the time factor, in this case it is observed an interesting feature such as the decoherence effect in the collapse and revival phenomenon of the Rabi oscillations.

In the Lamb-Dicke regime, $\eta_{j}<<1$, equation (11), can be well approximated by expanding the exponential terms up to second order in $\eta\left(\eta=\eta_{1}=\eta_{2}\right)$,

$$
\hat{H}_{i n}=\hbar \lambda(t) \hat{a}_{1}^{\dagger} \hat{a}_{2} \hat{\sigma}_{-}+H . c,
$$

where $\lambda(t)=-\varepsilon(v t) \wp^{0} \eta^{2} \exp \left(-\frac{\eta^{2}}{2}\right)$. In what follows, we will find the solution of the equations of motion for the operators $\hat{a}_{i}(t), \hat{\sigma}_{ \pm}(t)$ and $\hat{\sigma}_{z}(t)$ in the Heisenberg picture. Therefore the equations of motion related to the 
Hamiltonian (8) can be written as

$$
\begin{aligned}
\frac{d \hat{a}_{1}}{d t} & =-i \omega_{1} \hat{a}_{1}-i \lambda(t) \hat{a}_{2} \hat{\sigma}_{-}, \\
\frac{d \hat{a}_{2}}{d t} & =-i \omega_{2} \hat{a}_{2}-i \lambda(t) \hat{a}_{1} \hat{\sigma}_{+} \\
\frac{d \sigma_{-}}{d t} & =-i \omega_{0} \sigma_{-}+i \lambda(t) \hat{a}_{1} \hat{a}_{2}^{\dagger} \sigma_{z}, \\
\frac{d \sigma_{z}}{d t} & =2 i \lambda(t)\left(\hat{a}_{1}^{\dagger} \hat{a}_{2} \hat{\sigma}_{-}-\hat{a}_{1} \hat{a}_{2}^{\dagger} \hat{\sigma}_{+}\right) .
\end{aligned}
$$

After straightforward calculations, one can find an analytic time dependent solution as

$$
\begin{aligned}
\hat{a}_{1}(t)= & e^{i \hat{\mu} \alpha(t)}\left(\cos \left(\gamma_{1} \alpha(t)\right)-\frac{i \hat{\mu}}{\gamma_{1}} \sin \left(\gamma_{1} \alpha(t)\right)\right) \hat{a}_{1}(0) \\
& -\frac{i}{\gamma_{1}} e^{-i \omega_{1} t} e^{i \hat{\mu} I(t)} \sin \left(\gamma_{1} \alpha(t)\right) \hat{a}_{2}(0) \hat{\sigma}_{-}(0), \\
\hat{a}_{2}(t)= & e^{-i \omega_{2} t} e^{i \hat{\mu} \alpha(t)}\left(\cos \left(\gamma_{2} \alpha(t)\right)-\frac{i \hat{\mu}}{\gamma_{2}} \sin \left(\gamma_{2} \alpha(t)\right)\right) \hat{a}_{2}(0) \\
& -\frac{i}{\gamma_{2}} e^{-i \omega_{2} t} e^{i \hat{\mu} \alpha(t)} \sin \left(\gamma_{2} \alpha(t)\right) \hat{a}_{1}(0) \hat{\sigma}_{+}(0), \\
\hat{\sigma}_{-}(t)= & -\frac{i}{\gamma_{3}} e^{-i \omega_{12} t} e^{i \hat{\mu} \alpha(t)} \sin \left(\gamma_{3} \alpha(t)\right) \hat{a}_{1}(0) \hat{a}_{2}^{\dagger}(0) \\
& +e^{-i \omega_{12} t} e^{i \hat{\mu} \alpha(t)}\left(\cos \left(\gamma_{3} \alpha(t)\right)\right. \\
& \left.+\frac{i \hat{\mu}}{\gamma_{3}} \sin \left(\gamma_{3} \alpha(t)\right)\right) \hat{\sigma}_{-}(0), \\
\hat{\sigma}_{z}(t)= & \cos \left(2 \gamma_{4} \alpha(t)\right) \hat{\sigma}_{z}(0)+\frac{i}{\gamma_{4}} \sin \left(2 \gamma_{4} \alpha(t)\right) \\
& \times\left(\hat{a}_{2}^{\dagger} \hat{a}_{1} \hat{\sigma}_{+}(0)-\hat{a}_{1}^{\dagger} \hat{a}_{2} \hat{\sigma}_{-}(0)\right),
\end{aligned}
$$

where $\omega_{12}=\omega_{1}-\omega_{2}$,

$$
\begin{aligned}
\gamma_{1} & =\left(\hat{l}+\frac{1}{2}\right)\left(\hat{m}+\frac{3}{2}\right), \quad \gamma_{2}=\left(\hat{l}+\frac{3}{2}\right)\left(\hat{m}+\frac{1}{2}\right), \\
\gamma_{3} & =\left(\hat{l}-\frac{1}{2}\right)\left(\hat{m}+\frac{3}{2}\right), \quad \gamma_{4}=\left(\hat{l}+\frac{1}{2}\right)\left(\hat{m}+\frac{1}{2}\right), \\
\alpha(t) & =\int_{0}^{t} \lambda\left(t^{\prime}\right) d t^{\prime}, \quad \hat{\mu}=\left(\hat{a}_{1}^{\dagger} \hat{a}_{2} \hat{\sigma}_{-}+\hat{a}_{2}^{\dagger} \hat{a}_{1} \hat{\sigma}_{+}\right) .
\end{aligned}
$$

Having obtained the dynamical operators, we are therefore in a position to discuss some statistical properties of the system.

\section{Atomic inversion}

The entanglement of motional degrees of freedom of the center of mass of the ion with it's internal degrees of freedom manifests itself in the well known collapse and revival of population inversion 36 . We shall start with the atomic inversion from which we can discuss the collapse and revival phenomenon. The (internal level) ionic dynamics depend on the distributions of initial excitations of both the field and the center-of- mass vibrational motion, given by $\left\langle n\left|\rho_{f}(0)\right| n\right\rangle=\rho_{n n}^{f}(0)$ and $\left\langle m\left|\rho_{v}(0)\right| m\right\rangle=$ $\rho_{m m}^{v}(0)$, respectively. For instance, the atomic population inversion may be written as

$$
\left\langle\sigma_{z}(t)\right\rangle=\operatorname{Tr}\left(\sigma_{z}(0) \rho(0)\right)
$$

that is the difference between the probability of finding the system in the ground state and the probability of finding the system in the excited state.

To analyze the effects resulting from variation in the parameter $\lambda(t)$ on the atomic inversion we have plotted in figure 4 the function $\left\langle\sigma_{z}(t)\right\rangle$ against the scaled time $\lambda t$, for different values $\lambda(t)$, where $\lambda(t)=\lambda$ for figure (4a) and $\lambda(t)=\lambda \sinh (\varpi t)$ for figure $(4 \mathrm{~b})$, where $\varpi$ is a positive integer. In this figure let us take the parameters $\xi=10, q=1$ and $\phi=\pi / 2$, keeping in mind we are dealing with the problem at exact resonances. In figure (4a) we find that the value of the atomic inversion decreases from the maximum to its minimum, (approximately -0.75$)$ then it collapses, but then it starts to fluctuate around zero for a short period of time. Thus 

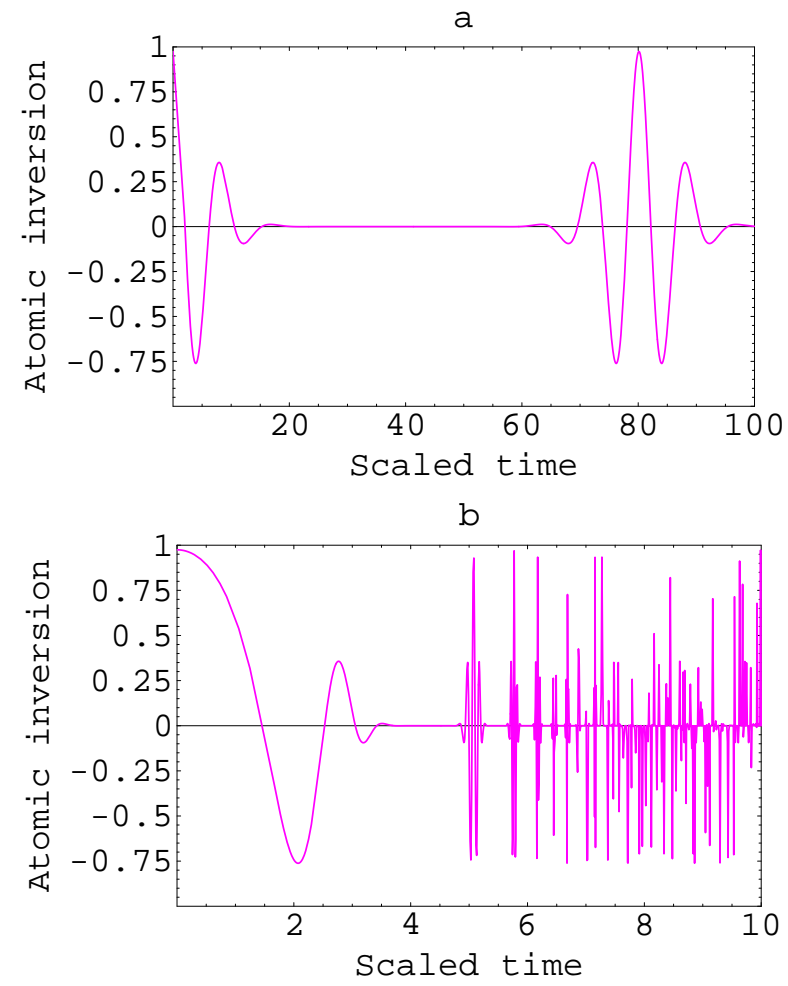

Fig. 4 The atomic inversion as a function of the scaled time $\lambda t$. Parameters: $\xi=10, q=1, \phi=0$, where (a) $\lambda(t)=\lambda t$ and (b) $\lambda(t)=\lambda \sinh (\varpi t)$

the Rabi frequencies become commensurate so that the atomic inversion has an exact periodic evolution with a scaled $\lambda$-dependent period. In this case there is no oscillations in the collapse region, as shown in figure 4a. A different pattern for the oscillations occurs if the number difference is slightly changed. Strictly speaking, when we consider the time dependent modulating function $\lambda(t)=\lambda \sinh (\varpi t)$, the function $\sinh (\varpi t)$ oscillates (however their period of oscillation is not constant) then the appearance of the collapses and revivals in almost a periodic way has been washed out in this case. On the other hand and in the presence of the time-dependent modulating function we find the behavior of the atomic inversion function is drastically changed where we can see irregular collapses and revivals occur, (see figure 4b). The short time revivals are strongly suppressed, as we see in figure $4 \mathrm{~b}$. We may compare the atomic inversion in both figure $3 \mathrm{a}$ and $3 \mathrm{~b}$, in the former case the collapse time is long enough to still allow revivals, while in the latter a shorter collapse time with the same amplitudes of the revivals.

It is interesting to explore to what conditions can be considered to generate a long living entanglement. This will be seen in the next section.

\section{Entanglement}

It is well known that entanglement between several particles is the most important feature of many quantum communication and computation protocols. In order to get a feel for how the entanglement of ion-field interaction affected by the presence of pair cat states we began by studying the aforementioned system that is analytically solvable and start from a factored initial state of both the ion and field. We consider the notion of entanglement entropy, that is, considering a quantum system in a pure state with the density matrix of the remaining space as $\rho_{A}=\operatorname{Tr}_{F}(\rho)$. The density matrix contains all information of any system in a mixed or pure state, and computing vital physical information on any such system is mostly determined by the eigenvalues of the density matrix. The partial entropy of entanglement for a bipartite pure state is defined as Von Neumann entropy of the 
reduced state,

$$
S_{A}=-\operatorname{Tr}\left\{\rho_{A} \log _{2} \rho_{A}\right\}
$$

This procedure is well established, and works well for all cases where the initial state of the system is in a pure state. Most work has been focused on this entanglement in time-independent modulating function, but we will focus on a time-dependent case.

On the other hand, the quantum entropy of the field $S_{f}(t)$ can be expressed in terms of the eigenvalues $\lambda_{F}^{ \pm}(t)$ of the reduced field density operator as follows

$$
S_{f}(t)=-\left[\lambda_{f}^{+}(t) \ln \lambda_{f}^{+}(t)+\lambda_{f}^{-}(t) \ln \lambda_{f}^{-}(t)\right] .
$$

In the case of a disentangled pure joint state $S_{f}(t)$ is zero, and for maximally entangled states it gives $\ln 2$ [20]. Due to the higher dimensionality of the problem we cannot obtain a simple analytical expression for equation (20), therefore the numerical approach becomes indispensable.

Now let us discuss the behavior of the field entropy resultant of the existence of the time dependent modulating function $\lambda(t)$. Figure 5 is a plot of quantum field entropy $S_{f}(t)$ as a function of scaled time parameter $\lambda t$ for $\eta=0.2, \lambda(t)=\lambda$ and considering different values of $\xi$ where $\xi=10$ for figure $5 \mathrm{a}$ and $\xi=20$ for figure $5 \mathrm{~b}$. Our numerical analysis was performed using other parameters from recent experiments 23 37, where a trapped ${ }^{9} \mathrm{Be}^{+}$ion was laser-cooled to the zero-point energy and then prepared in a superposition of spatially separated coherent harmonic oscillator states. In 38 there have
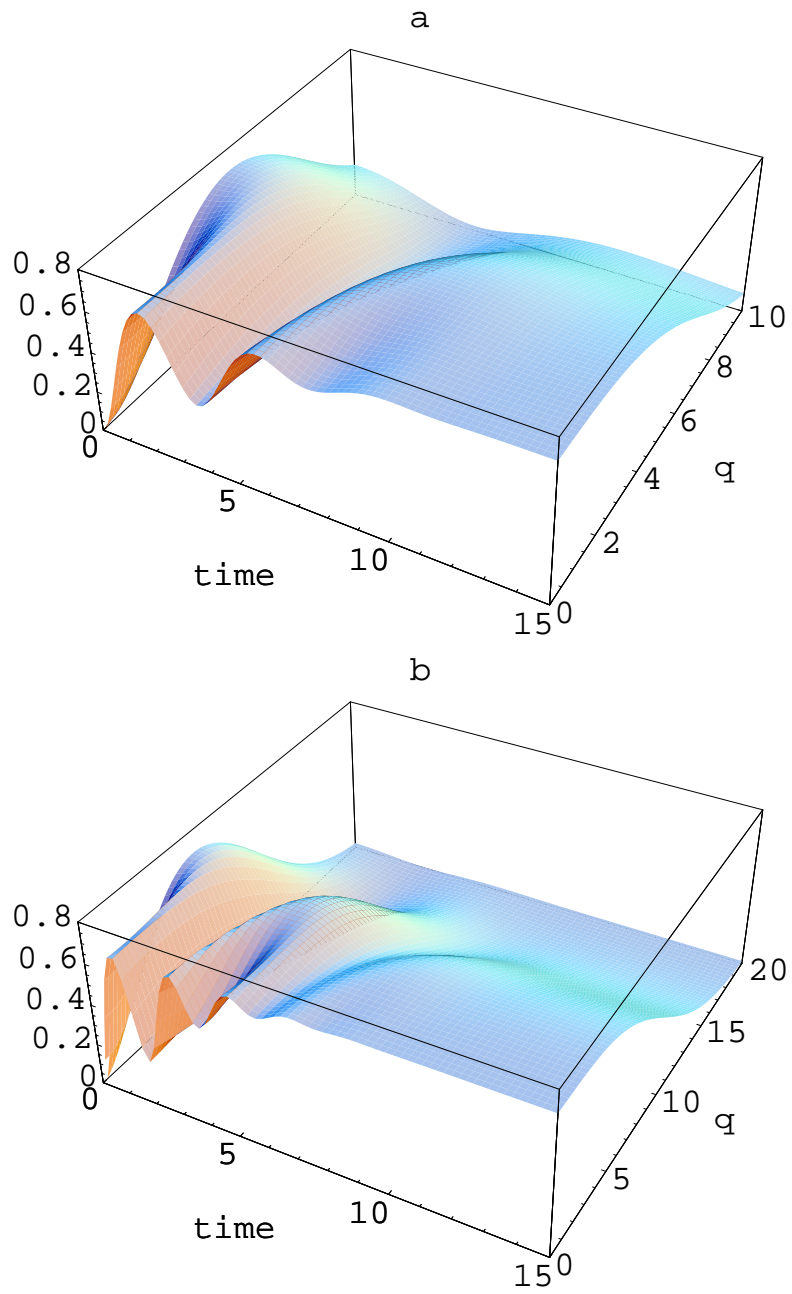

Fig. 5 The quantum field entropy $S_{f}(t)$ as a function of the scaled time $\lambda t$. The parameters are $q=1, \eta=0.2, \lambda(t)=\lambda$ and different values of $\xi$ where (a) $\xi=10$ and (b) $\xi=20$.

been recent demonstrations of the coupling between cavities and single-ion traps, where, a single calcium ion is stored in a spherical trap and placed in the center of a confocal resonator.

It is interesting to note that, the maximum entanglement is decreases as the parameter $q$ increased (see figure 5a). As we see from figure $5 \mathrm{a}$, as time goes on we note a growth in $S_{f}(t)$, followed by a sudden decrease, almost down to zero at large values of $q$. When the time in- 
creases further we see that the entanglement has a long surviving, if we keep smaller values of $q$. The constat value of $S_{f}(t)$ is interpreted as a result of quantum entanglement between ion and fields, surviving for a long time interaction. However, an interesting situation may arise when the parameter $\xi$ takes large values (say 20 in figure 5b). In this case, for some small fixed values of $q$, the entanglement surviving starts at earlier time. However, the overall features of the process are similar in both small and large values of $\xi$. An important difference occurs only at earlier interaction time, where the number of oscillations is increased with increasing $\xi$. Also, the amplitudes of these oscillations are bigger compared to the case in which $\xi=10$ (see figures $5 \mathrm{a}$ and $5 \mathrm{~b}$ ). As seen in the entropy plot in figure $5 \mathrm{~b}$, the pure state is basically obtained for large values of the parameter $q$ (because the natural scale of $q$ is between 0 and $\xi$, in figure $5 \mathrm{~b}$, we expand the scale of $q$ to take values between 0 and 20).

On the contrary, as can be seen from figure 5, if the fields are initially prepared in a pair cat state and the modulating function is taken to be time-dependent $\lambda(t)=\lambda \sinh (\varpi t)$, then the entanglement parameter is significantly larger than zero for any time $t>0$, but with some oscillations from time to time. Also, the number of oscillations is increased as the parameter $q$ decreased associated with low entanglement reaches zero for smaller values of the parameter $\xi$ (see figure 6 ).
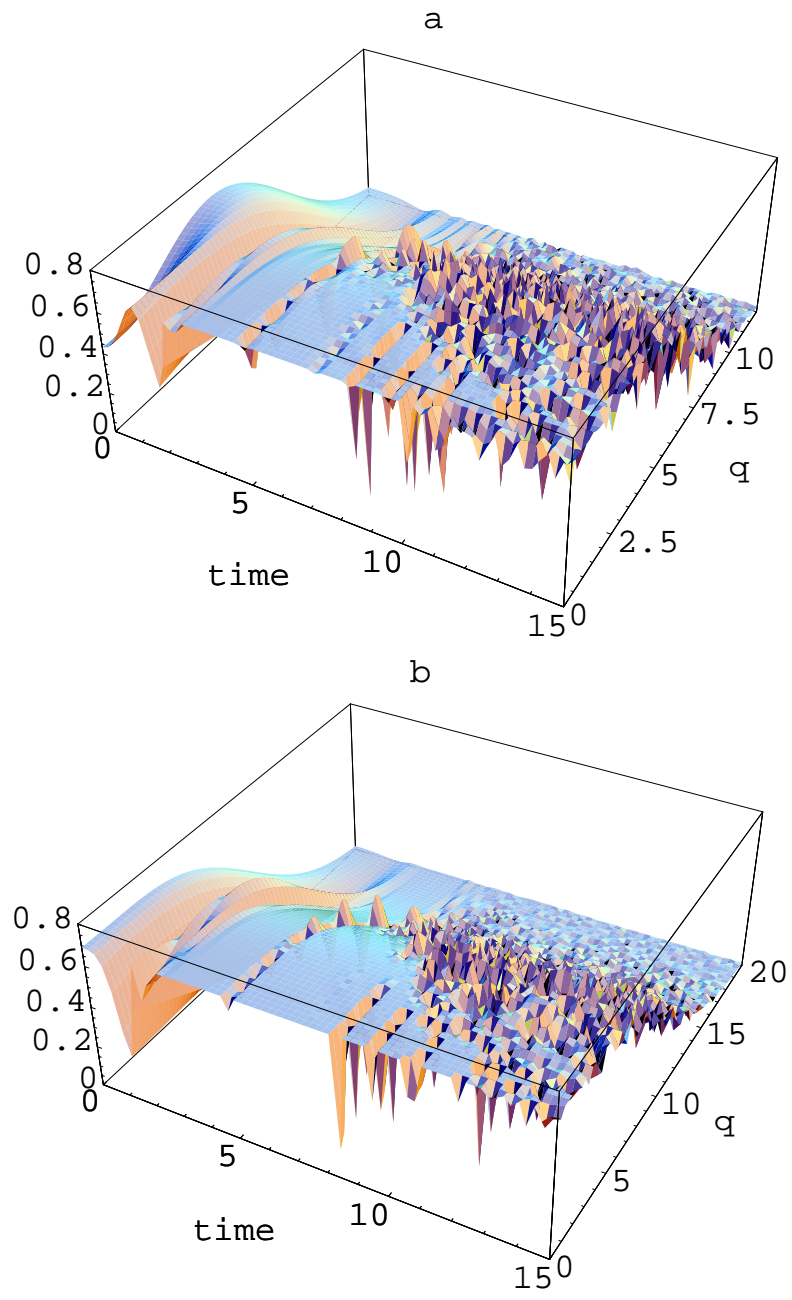

Fig. 6 The same as figure 5 but $\lambda(t)=\lambda \sinh (\varpi t)$.

Another interesting aspect is that the time-dependent modulating function $\lambda(t)$ has an important affect on the entanglement, where, the total ion-field state can not have its purity diminished, which means that as the field becomes more pure the ionic state must be closer to a mixed state. Also, in this case, it seems remarkable that entanglement survive for long time but some oscillations occur here. More surprisingly still, survive is long-lived for the time-independent case. We should pointed out that if we simply use a time-independent modulating function, we would expect entanglement to survive. 
Practically speaking, as can be deduced from figure $5 \mathrm{a}$, the oscillations in degree of entanglement between the ion and the field quickly damp out with an increase in $q$. The subsystem will not disentangle from each other until the steady state is reached as long as the interaction is present. This means that neither the ion nor the field will return back to a pure state except at the beginning of the interaction and $t \rightarrow \infty$, contrast to the $q=0$ case. In order to gain insight into the general behavior we have considered different values of $\xi$ in figure $6 \mathrm{~b}$. The direct comparison of figure $6 \mathrm{a}$ and figure $6 \mathrm{a}$, the amplitude of the oscillations in the degree of entanglement is increased with increasing the parameter $\xi$.

The remaining task is to identify and compare the results presented above for the entanglement degree with another accepted entanglement measure such as the linear entropy. The question of the ordering of entanglement measures was raised in reference 39]. It was proved that all good asymptotic entanglement measures are either identical or fail to uniformly give consistent orderings of density matrices 40. The best understood case, not surprisingly, is the simplest. In order to better characterize the subsystems, we may refer here to another measure of the entanglement of a reduced density matrix which is the product state identification

$$
S_{L}=1-\operatorname{Tr} \rho_{A}^{n}, \quad n \geq 2,
$$

which is zero for a product state, and unity for a maximally entangled state. This measure is equivalent to the
Rĕnyi entropy 12 and is not well suited for much more than to single out a pure state, as with increasing $n$ any entangled state will converge to zero in this measure.

In figure 7 we plotted the entanglement according to a high order linear entropy and compared with the von Neumann entropy. One, possibly not very surprising, principal observation is that the numerical calculations corresponding to the same parameters, which have been considered in figures 4 and 5 , gives nearly the same behavior (see figure 7). This means that both the entanglement due to the quantum field entropy $S_{f}$ and high order linear entropy $S_{L}$ measures are qualitatively the same. The important consequence of this observation is that the use of quantum entropy and linear entropy as measures of entanglement are equivalent. We must stress, however, that no single measure alone is enough to quantify the entanglement in a multi-partite systems. Finally, we may say that, it is possible to obtain a long living entanglement using the time-independent modulating function in pair cat state. This result is quit surprising, since the previous studies [20] in the entanglement for the time-independent interaction for the initial coherent state does not contain this interesting feature. Which means that the pair cat state as an initial state of the field plays very important roles in the quantum information process.

A proposal for the generation of motional pair cat states in a two-dimensional anisotropic trap has been introduced in Ref. 41]. In order to generate the pair cat 


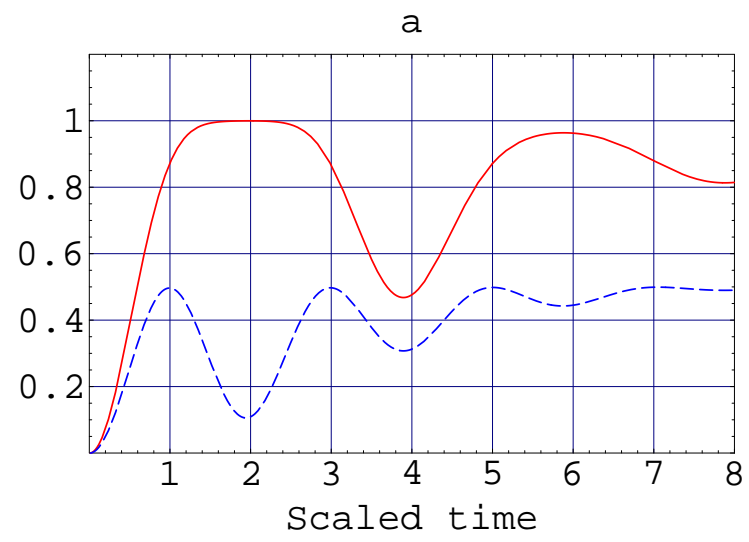

b

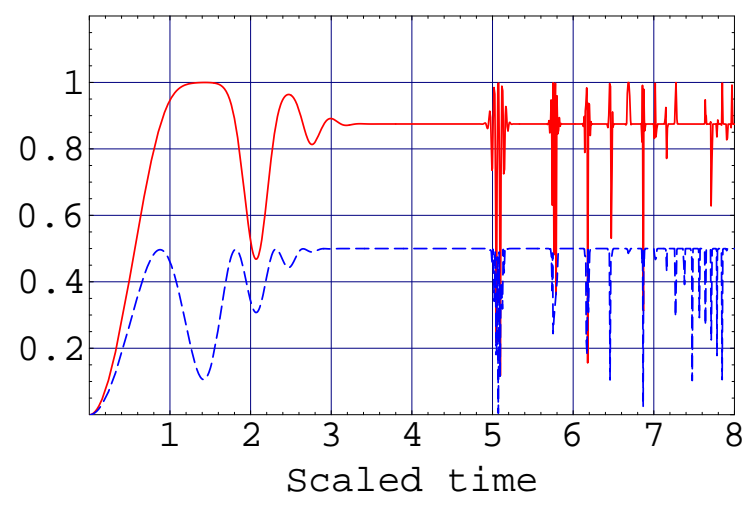

Fig. 7 Time evolution of entanglement degree $S_{L}$ as a function of the scaled time $\lambda t$. The parameters are the same as in figure 3 , where $n=2$ (the solid curve) and $n=3$ (the dotted curve).

states for the motion in a two-dimensional anisotropic trap we require two laser beams and the frequencies of the laser beams are chosen as $\omega_{1}=\omega_{0}-2 \nu_{x}-2 \nu_{y}$, and $\omega_{2}=\omega_{0}$. The generation method requires a sequence of many measurements of the internal electronic state at different time instants appropriately chosen [2]. Apart from detecting quantum entanglement in these states, might be a useful way to fully characterize simple quantum logic gates 42 .

\section{Summary}

The main results of this work are summarized as follows: After using the Heisenberg approach to the quantum operator, we have investigated effects produced by the ion-field interaction. We have shown that the input pair cat states exhibit strong nonclassical features due to the correlation between the two modes. The phenomenon of quantum revivals in the time-dependent ion-field interaction and the atomic inversion properties as well as the entanglement have been analyzed. Also, it has been shown that the atomic inversion as a function of the scaled time may display different structures of beats depending on the initial state of the field and the vibrational motion as well as on the time-dependent interaction. Effects such as suppression or attenuation of the Rabi oscillations and long time scale revivals as well as a periodic dynamics have been observed.

Using pair cat states as an initial state of the fields, our analysis shows that the fluctuations of the entanglement between maximum and minimum values are irregular for both the time-independent and time-dependent cases at a short period of the interaction time. Our results reveal a new feature in the entanglement as a function of the scaled time, that is a long living entanglement. This feature depends on the parameters $\xi$ and $q$. These interesting features may pave the way to quantum information applications such as deterministic all-optical quantum computation. The results can be relevant for 
basic studies on entanglement as well as for applications in the preparation and manipulation of quantum states for quantum information purposes. As far as scalability is concerned, one could think in having several trapped ions in a high-Q cavity, exchanging information via the cavity photons, or other more sophisticated schemes involving trapped ions, phonons and photons.

It is to be remarked that, the entropy of entanglement can be determined indirectly by experiment using the quantum state tomography [11. This fact comes from the definition of the entropy of entanglement which is associated with the density matrix of quantum state.

We believe that the pair cat states may be a useful tool to be considered in obtaining a long living entanglement of systems of indistinguishable particles. While our analysis was carried out with respect to the example of a single trapped ion beyond Lamb-Dicke regime, we emphasize that the results here reported apply equally well to any bipartite system. We hope to report on such issues in a forthcoming paper.

\section{ACKNOWLEDGMENTS}

The author would like to thank the referees for their objective comments that improved the text in many points. Also, it is a pleasure to thank G. Gour, A.-S. F. Obada, S. S. Hassan, and M. S. Abdalla for inspiring discussions.

\section{References}

1. R. Blatt, Entangled World, ed. Jrgen Audretsch, (WileyVCH, Weinheim 2006) pp. 235-270; S. Gulde, et al., in
Atomic Physics 18 (Proceedings of the ICAP 2002).

2. S. Maniscalco, J. Opt. B: Quantum Semiclass. Opt. 7, R1 (2005); F. L. Semiao, A. Vidiella-Barranco, and J. A. Roversi, Phys. Rev. A 66, 063403 (2002).

3. G. P. T. Lancaster, H. Häffner, M.A. Wilson, C. Becher, J. Eschner, F. Schmidt-Kaler, R. Blatt, Appl. Phys. B: Lasers \& Optics, 76, 805 (2003)

4. D. F. V. James, Appl. Phys. B: Lasers \& Optics, 66, 181 (1998).

5. D. J. Wineland, C. Monroe, W. M. Itano, B. E. King, D. Leibfried, C. Myatt and C. Wood, Physica Scripta. Vol. T76, 147, (1998); B.E. King et al., Phys. Rev. Lett. 81, 1525 (1989).

6. M. Keller, B. Lange, K. Hayasaka, W. Lange, H. Walther, Appl. Phys. B: Lasers \& Optics, 76, 125 (2003)

7. M. Sasura and V. Buzek, J. Mod. Opt. 49, 1593 (2002); A. Messina, S. Maniscalco and A. Napoli, J. Mod. Opt. 501 (2003).

8. M. Abdel-Aty, Appl. Phys. B: Laser \& Optics, 81, 193 (2005)

9. M. Abdel-Aty, J. Phys. A: Math. Gen. 38, 8589 (2005)

10. F. Schmidt-Kaler, H. C. Ngerl, D. Leibfried, R. Blatt, M. Brune, J. M. Raimond, S. Haroche, in The Physics of Quantum Information, (Springer, Berlin, 2000).

11. M. A. Nielsen and I. L. Chuang, Quantum Computation and Quantum Information (Cambridge University Press, Cambridge, UK, 2000); M. Popp, F. Verstraete, M.A. Martin-Delgado and I. Cirac, Appl. Phys. B: Lasers \& Optics 82, 225 (2006).

12. S. O. Skroseth and K. Olaussen, Phys. Rev. A 72, 022318 (2005); B.-Q. Jin and V. E. Korepin, J. Stat. Phys. 116, 79 (2004); S. O. Skrvseth, arXiv:quant-ph/0508160 
(2005); M. Ziman and V. Buzek, Phys. Rev. A 73, 012312 (2006)

13. C. H. Bennett, H. J. Bernstein, S. Popesu, and B. Schumacher, Phys. Rev. A 53, 2046 (1996).

14. P. Horodecki, R. Horodecki, M. Horodecki, Acta Phys. Slov. 48, 141 (1998).

15. V. Vedral, M. B. Plenio, K. Jacobs, and P. L. Knight, Phys. Rev. A 56, 4452 (1997).

16. V. Vedral, M. B. Plenio, M. A. Rippin, and P. L. Knight, Phys. Rev. Lett. 78, 2275 (1997).

17. V. Vedral and M. B. Plenio, Phys. Rev. A 57, 1619(1998).

18. R. Guo and H. Guo, Phys. Rev. A 73, 012103 (2006); S. Furuichi and M. Ohya, Lett. Math. Phys. 49 , 279 (1999).

19. S. Furuichi and M. Abdel-Aty, J. Phys. A: Math. \& Gen. 34, 6851 (2001);

20. S. J. D. Phoenix and P. L. Knight, Ann. Phys. (N. Y) 186, 381 (1988); S. J. D. Phoenix and P. L. Knight, Phys. Rev. A 44, 6023 (1991); Phys. Rev. Lett. 66, 2833 (1991).

21. M. Brune, S. Haroche, J. M. Raimond, L. Davidovich and N. Zagury, Phys. Rev. A 45, 5193 (1992); V. Buzek and P.L. Knight, Quantum Interference, Superpositions States of Light and Nonclassical Effects, in Progress in Optics 34 (1995) 1, Ed. E. Wolf (Elsevier, Amsterdam, 1995).

22. C. C. Gerry and P. L. Knight, Quantum Superpositions and Schrödinger Cat States in Quantum Optics, Am. J. Phys. 65, 964 (1997).

23. C. Monroe, D. M. Meekhof, B. E. King, and D. J. Wineland, Science 272, 1131 (1996).
24. S. C. Gou, J. Steinbach and P. L. Knight, Phys. Rev. A54, R1014 (1996).

25. S. C. Gou, J. Steinbach and P. L. Knight, Phys. Rev. A54, 4315 (1996).

26. C. C. Gerry, S. C. Gou and J. Steinbach, Phys. Rev. A55, 630 (1998).

27. D.J. Wineland, C. Monroe, W.M. Itano, D. Kielpinski, B. E. King, C. J. Myatt, Q. A. Turchette, and C. S. Wood, "Quantum Computation, Spectroscopy of Trapped Ions, and Schrdinger's Cat," in Quantum Coherence and Decoherence, Proceedings of the 6th International Symposium on Foundations of Quantum Mechanics in the Light of New Technology (ISQM-Tokyo '98), edited by Y. A. Ono and K. Fujikawa (Elsevier Science, Amsterdam, 1999), pp. 103-108.

28. H. Jeong and M. S. Kim, Phys. Rev. A 65, 042305 (2002).

29. P. T. Cochrane, G. J. Milburn, and W. J. Munro, Phys. Rev. A 59, 2631 (1999).

30. J. R. Klauder and B. Skagerstam, Coherent States: Applications in Physics and Mathematical Physics, World Scientific, Singapore, 1985.

31. G. S. Agarwal, J. Opt. Soc. Am. B 5, 1940 (1988)

32. G. S. Agarwal, Phys. Rev. Lett. 57, 827 (1986).

33. C. C. Gerry and R. Grobe, Phys. Rev. A 51, 1698 (1995)

34. G. S. Agarwal and A. Biswas, J. Opt. B: Quantum Semiclass. Opt. 7, $350(2005)$

35. C. A. Blockley, D. F. Walls and H. Risken, Europhys. Lett. 17, 509 (1992)

36. S. S. Sharma and N. K. Sharma, J. Phys. B 35, 1643 (2002)

37. D. M. Meekhof, C. Monroe, B. E. King, W. M. Itano, D. J. Wineland, Phys. Rev. Lett. 76, 1796 (1996). 
38. A. B. Mundt, A. Kreuter, C. Becher, D. Liebfried, J. Eschner, F. Schmidt-Kaler and R. Blatt, Phys. Rev. Lett. 89, 103001 (2002).

39. J. Eisert, M.B. Plenio, J. Mod. Opt. 46, 145 (1999)

40. S. Virmani, M.B. Plenio, Phys. Lett. A 268, 31 (2000)

41. S.-B. Zheng, J. Opt. B: Quantum Semiclass. Opt. 3, 298 (2001)

42. J. F. Poyatos, J. I. Cirac and P. Zoller, Phys. Rev. Lett. $78,390(1997)$ 